\title{
Genetic Variability, Trait Association and Path Analysis in Cowpea (Vigna unguiculata L. Walp)
}

\author{
B. Nandini Priya Yadav* and Duddukur Rajasekhar \\ Department of Genetics and Plant Breeding, Naini Institute of Agriculture, \\ SHUATS, Prayagraj (Allahabad), Uttar Pradesh, India \\ *Corresponding author
}

\section{A B S T R A C T}

\begin{tabular}{|l|}
\hline Key w o r d s \\
Cowpea, Genetic \\
Variability, \\
Correlation, Path \\
analysis
\end{tabular}

\section{Introduction}

Cowpea (Vigna unguiculata (L.) Walp) also known as long yard bean, lobia. Cowpea is also known as vegetable meat. It is a self-
The study was undertaken on the 30cowpea genotypes along with one check (Pant lobia-5) to study the genetic variability, correlation and path analysis at Field Experimentation Centre, Department of Genetics and Plant Breeding, Sam Higginbottom University of Agriculture, Technology and Sciences(SHUATS), Prayagraj during Kharif 2016 in Randomized Block Design with three replications. Analysis of variance showed highly significant differences among $(29+1$ check) cowpea genotypes for 13 yield contributing characters studied. Maximum genotypic and phenotypic variance was recorded for plant height, days to maturity, pods per plant and biological yield. Maximum GCV and PCV were recorded for pods per plant, primary branches per plant and pod length. High heritability coupled with high genetic advance as percent of mean was recorded for pods per plant, primary branches per plant and plant height. The genotypic and phenotypic correlation coefficients were computed among 13 characters' days to 50\% flowering, days to $50 \%$ pod setting, pods per plant, 100 seed weight, harvest index and biological yield showed highly significant positive association with seed yield at both phenotypic and genotypic levels. While plant height is significant negative correlation with the seed yield at both phenotypic and genotypic levels. Path values revealed that days to $50 \%$ flowering, pods per plant, clusters per pod, harvest index, biological yield showing direct positive effect on seed yield at both phenotypic and genotypic levels. Primary branches per plant showed direct and negative effect on seed yield at both phenotypic and genotypic levels. Pod length,100 seed weight, showed direct and positive effect on seed yield at genotypic level. Days to 50\% maturity, days to 50\% pod setting, plant height, seeds per pod showed direct positive effect on seed yield at phenotypic level. 
and sub-tropics. Green pod of cowpea contains $85 \%$ moisture, $3.0 \%$ protein, $1.0 \%$ minerals, $2.0 \%$ fiber, $8.0 \%$ carbohydrates, $0.72 \%$ calcium, $0.59 \%$ phosphorus, $0.2 \% \mathrm{mg}$ iron, $0.009 \%$ riboflavin and $0.007 \%$ thiamin (Anonymous, 2011). It is the only pulse crop rich in lysine (Hazara and Som, 1999). The optimum temperature for growth and development is around $30^{\circ} \mathrm{C}$. It is a heat and drought-tolerant crop. It grows best during summer. It is also grown on a wide range of soils but the crop performs better in sandy soils, as it tends to be less restrictive to root growth. It is more tolerant to infertile and acid soils than many other crops. It is usually grown under dryland rather than irrigated conditions. The magnitude of genetic variance denotes how much of the variability of the characters is heritable advance can be achieved. Hence, collection, maintenance and evolution of germplasm for studying genetic variability of economically important traits is one of the basic steps for initiating breeding programme for genetic improvement of cowpea. The genetic variability of cowpea is high for almost all important agronomic traits, but in comparison with other crops little breeding work has been done and focused mainly on the improvement of yield and increased disease resistance (Hall et al., 2002).For the improvement in any crop species, the knowledge of genetic variability for characters of economic importance and their heritability and genetic advance is of utmost importance in planning future breeding programme (Singh et al., 2007). Direct selection for the yield is not much effective as quantitative characters are controlled by polygenes. Hence, knowledge about the association of various traits which will directly or indirectly contribute to yield is crucial. Correlation coefficients explain the degree of association among the characters. However, it is difficult to explain a system of correlation when the indirect association between the characters increases. The method of path coefficient analysis development by Sewall wright (1921) is helpful in partitioning correlation into direct and indirect effects and in the assessment of relative contributions of each component to the yield. Heritability of a character is important for the cowpea breeder because it provides him an idea of the extent of genetic control for the expression of a particular character (Chopra, 2000). Moreover, heritability serves as a guide to the reliability of phenotypic variability in the selection programme (Hamdi, 1992). The major function of heritability estimates is to provide information on degree of transmission of traits from the parents to the progeny. Such estimates facilitate evaluation of hereditary and environmental effects in phenotypic variation, thereby aiding in selection. Estimates of heritability can be used to predict genetic advance under selection so that the breeder can anticipate improvement from different types and intensities of selection. Yield is a complex entity and depends on the expression of a number of traits known as yield components. The objective of the present study is to investigate the genetic variability, heritability and genetic advance of yield and its attributing characters, study correlation between yield and its attributing parameters and estimate direct and indirect effect of yield contributing characters on seed yield.

\section{Materials and Methods}

In the present investigation, 30 genotypes were grown in the season Kharif, 2017 at the Field Experimentation Centre, Department of Genetics and Plant Breeding, Sam Higginbottom University of Agriculture, Technology and Sciences, Allahabad (U.P). The experimental materials consist of 30 cowpea genotypes obtained from IIVR Varanasi and Research station, Pantnagar. The experiment was laid out in Randomized complete block design with three replications the genotypes were sown by hand dibbling in 
each plot by imposing randomization in each replication along with check Pant lobia-5. In each plot the spacing used is $45 \times 10 \mathrm{~cm}$. The fertilizer dose 25:40:25 NPK Kg/ha and all the recommended dose were followed to raise a good crop. Data was recorded on five randomly selected competitive plants per replication for 13 yield components plant height $(\mathrm{cm})$, number of primary branches per plant, days to $50 \%$ flowering, days to $50 \%$ pods setting, number of clusters per plant, number of pods per plant, days to maturity, pod length $(\mathrm{cm})$, biological yield $(\mathrm{g})$, number of seeds per pod, hundred seed weight $(\mathrm{g})$, seed yield per plant $(\mathrm{g})$, harvest index $(\%)$. The statistical analysis and variance due to different sources was worked out according to Fisher, 1936 Analysis of Variance, Heritability (Burton and Devane, 1953), Genetic Advance (Johnson et al., 1955), Genotypic and phenotypic correlation (Al Jibouri et al., 1958) and Path coefficient analysis (Dewey and Lu, 1959)

\section{Results and Discussion}

Analysis of variance was carried out for 13 characters in cowpea genotypes and the results are presented in Table 1 . The variance due to treatment was highly significant for all thirteen characters studied. This gives the evidence of magnitude of genetic variability among genotypes were differed significantly. Estimation of components of genetic parameters of variation for yield and its attributes exhibited wide range of genetic variation. The results indicated that PCV values were higher than GCV values for few characters which indicated impact of environment on the expression of various quantitative characters (Nigude et al., 2004). Maximum GCV and PCV were recorded for pods per plant, primary branches per plant, pod length and plant height, thus scope for genetic improvement through selection for these characters, similar findings was also reported by Pal et al., (2003).

Estimates of heritability are a good index for predicting the transmission of character from parents to their offspring (Falconer, 1981). Biological yield (97\%), seed yield (95\%), pods per plant (94\%), 100 seed weight (93\%), pod length (93\%), days to $50 \%$ flowering (82\%) and days to $50 \%$ pod setting $(92 \%)$ showed high heritability.

Heritability alone provides no indication of the amount of genetic improvement that would result from selection of individual genotypes. Hence knowledge about genetic advance coupled with heritability is most useful.

Genetic advance is the improvement in the mean of selected families over the base population (Lush, 1949 and Johnson and Robinson, 1955). Number of pods per plant $\left(\mathrm{h}^{2}=94\right.$, GAM $\left.=79.50\right)$, number of primary branches per plant $\left(\mathrm{h}^{2}=81, \mathrm{GAM}=53.02\right)$, plant height $\left(h^{2}=82, G A M=43.89\right), 100$ seed weight $\left(\mathrm{h}^{2}=93, \mathrm{GAM}=37.49\right)$ showed high heritability with high genetic advance (Table 2). These results suggest the preponderance of additive gene action in the expression of these characters and shows ample scope of selection.

The genotypic and phenotypic correlation coefficients were computed among 13 characters (Table 3) days to $50 \%$ flowering, days to $50 \%$ pod setting, pods per plant, 100 seed weight, harvest index and biological yield showed highly significant positive association with seed yield at both phenotypic and genotypic levels. While plant height showed significant negative association with seed yield at both phenotypic and genotypic levels. Days to maturity is significant positive association with the seed yield at genotypic level. 
Table.1 Analysis of variance for 13 different quantitative characters of 30 cowpea genotypes

\begin{tabular}{|c|c|c|c|c|c|c|c|c|c|c|c|c|c|c|}
\hline $\begin{array}{l}\text { Source of } \\
\text { variation }\end{array}$ & d.f. & $\begin{array}{c}\text { Days to } \\
50 \% \\
\text { flowerin } \\
\mathrm{g}\end{array}$ & $\begin{array}{c}\text { Days to } \\
\mathbf{5 0 \%} \\
\text { maturit } \\
y\end{array}$ & $\begin{array}{l}\text { Days to } \\
50 \% \text { pod } \\
\text { setting }\end{array}$ & $\begin{array}{c}\text { Plant } \\
\text { height }\end{array}$ & $\begin{array}{c}\text { Primar } \\
\text { y } \\
\text { branche } \\
\text { s/ plant }\end{array}$ & $\begin{array}{c}\text { Clusters/ } \\
\text { plant }\end{array}$ & $\begin{array}{l}\text { Pods/ } \\
\text { plant }\end{array}$ & $\begin{array}{c}\text { Pod } \\
\text { length }\end{array}$ & $\begin{array}{c}\text { Seeds/ } \\
\text { pod }\end{array}$ & $\begin{array}{c}\text { Seed } \\
\text { index }\end{array}$ & $\begin{array}{c}\text { Biological } \\
\text { yield/ } \\
\text { plant }\end{array}$ & $\begin{array}{c}\text { Harvest } \\
\text { index }\end{array}$ & $\begin{array}{l}\text { Seed } \\
\text { yield/ } \\
\text { plant }\end{array}$ \\
\hline Replications & 2 & 57.33 & 136.06 & 41.69 & 677.74 & 12.83 & 13.30 & 205.34 & 9.42 & 8.48 & 12.18 & 177.93 & 8.69 & 11.54 \\
\hline Treatments & 29 & 10.90 & 1.81 & 2.68 & 57.92 & 1.41 & 0.10 & 5.43 & 9.94 & 0.34 & 0.05 & 1.02 & 1.30 & 0.27 \\
\hline Error & 58 & 3.47 & 1.13 & 1.14 & 202.06 & 0.92 & 1.16 & 3.95 & 4.03 & 0.34 & 0.29 & 1.73 & 0.44 & 0.19 \\
\hline
\end{tabular}

Table. 2 Genetic parameters for 13 biometrical characters of 30 cowpea genotypes

\begin{tabular}{|c|c|c|c|c|c|c|c|c|}
\hline \multirow{2}{*}{$\begin{array}{l}\text { S. } \\
\text { No. }\end{array}$} & \multirow[t]{2}{*}{ Characters } & \multicolumn{2}{|c|}{ Range } & \multirow{2}{*}{$\begin{array}{l}\text { Grand } \\
\text { Mean }\end{array}$} & \multirow{2}{*}{$\begin{array}{l}\text { Phenotypic } \\
\text { Coefficient } \\
\text { of variance } \\
\text { (PCV) }\end{array}$} & \multirow{2}{*}{$\begin{array}{l}\text { Genotypic } \\
\text { Coefficient of } \\
\text { Variance } \\
\text { (GCV) }\end{array}$} & \multirow{2}{*}{$\begin{array}{c}\text { Heritability } \\
\text { (broad sense) } \\
(\%)\end{array}$} & \multirow{2}{*}{$\begin{array}{c}\text { Genetic } \\
\text { Advance } \\
\text { as } \\
\text { Percent mean }\end{array}$} \\
\hline & & Minimum & Maximum & & & & & \\
\hline 1 & Days to $50 \%$ Flowering & 31.66 & 46.66 & 39.33 & 10.77 & 11.77 & 84 & 20.31 \\
\hline 2 & Days to $50 \%$ Pod Setting & 51.66 & 66.66 & 57.92 & 6.33 & 6.61 & 92 & 12.56 \\
\hline 3 & Plant Height & 35.36 & 93.82 & 61.32 & 23.59 & 26.13 & 82 & 43.89 \\
\hline 4 & Primary Branches / Plant & 3.66 & 11.66 & 6.97 & 28.55 & 31.68 & 81 & 53.02 \\
\hline 5 & Clusters/ Plant & 12.00 & 22.00 & 15.36 & 13.09 & 14.84 & 78 & 23.78 \\
\hline 6 & Pods/ Plant & 9.00 & 44.00 & 20.63 & 39.71 & 40.83 & 94 & 79.50 \\
\hline 7 & Seeds/ Pod & 11.66 & 18.33 & 15.05 & 10.94 & 11.61 & 89 & 21.23 \\
\hline 8 & Pod Length & 12.56 & 19.53 & 15.83 & 39.71 & 46.66 & 39.33 & 79.50 \\
\hline 9 & Days of Maturity & 66.00 & 87.66 & 74.82 & 8.96 & 9.08 & 98 & 18.24 \\
\hline 10 & Seed Index & 7.56 & 15.08 & 10.56 & 18.85 & 19.52 & 93 & 37.49 \\
\hline 11 & Seed Yield/ Plant & 15.51 & 22.07 & 19.19 & 10.92 & 11.19 & 95 & 21.96 \\
\hline 12 & Biological Yield/ Plant & 77.97 & 109.27 & 93.31 & 8.21 & 8.33 & 97 & 16.68 \\
\hline 13 & harvest Index & 15.43 & 21.93 & 17.81 & 8.64 & 9.30 & 86 & 16.53 \\
\hline
\end{tabular}


Table.3 Correlation coefficient between yield and its related traits in 30 cowpea genotypesat both genotypic and phenotypic levels

\begin{tabular}{|c|c|c|c|c|c|c|c|c|c|c|c|c|c|c|}
\hline Characters & & $\begin{array}{c}\text { Days to } \\
50 \% \\
\text { Flowering }\end{array}$ & $\begin{array}{l}\text { Days to } \\
\text { 50\% Pod } \\
\text { Setting }\end{array}$ & $\begin{array}{l}\text { Plant } \\
\text { Height }\end{array}$ & $\begin{array}{c}\text { Days to } \\
\text { Maturity }\end{array}$ & $\begin{array}{c}\text { Primary } \\
\text { Branches/ } \\
\text { Plant }\end{array}$ & $\begin{array}{l}\text { Pods Per } \\
\text { Plant }\end{array}$ & $\begin{array}{l}\text { Pod Length } \\
\text { cm }\end{array}$ & $\begin{array}{l}\text { Seeds Per } \\
\text { Pod }\end{array}$ & $\begin{array}{c}\text { Clusters Per } \\
\text { Plant }\end{array}$ & $\begin{array}{c}100 \\
\text { Seed } \\
\text { Weight }\end{array}$ & $\begin{array}{l}\text { Biological } \\
\text { yield }\end{array}$ & $\begin{array}{l}\text { Harvest } \\
\text { Index }\end{array}$ & $\begin{array}{c}\text { Seed } \\
\text { yield } \\
\text { per } \\
\text { plant }\end{array}$ \\
\hline \multirow{2}{*}{$\begin{array}{c}\text { Days to } \\
50 \% \\
\text { Flowering }\end{array}$} & $\mathbf{G}$ & 1 & & & & & & & & & & & & \\
\hline & $\mathbf{P}$ & 1 & & & & & & & & & & & & \\
\hline \multirow{2}{*}{$\begin{array}{l}\text { Days to } \\
50 \% \text { Pod } \\
\text { Setting }\end{array}$} & $\mathbf{G}$ & $0.345^{* *}$ & 1 & & & & & & & & & & & \\
\hline & $\mathbf{P}$ & $0.337 * *$ & 1 & & & & & & & & & & & \\
\hline \multirow{2}{*}{$\begin{array}{c}\text { Plant } \\
\text { Height }\end{array}$} & G & $-0.188 \mathrm{NS}$ & $-0.337 * *$ & 1 & & & & & & & & & & \\
\hline & $\mathbf{P}$ & $-0.085 \mathrm{NS}$ & $0.264^{*}$ & 1 & & & & & & & & & & \\
\hline \multirow{2}{*}{$\begin{array}{l}\text { Days to } \\
\text { Maturity }\end{array}$} & $\mathbf{G}$ & $0.400 * *$ & $-0.746^{* *}$ & $-0.367 * *$ & 1 & & & & & & & & & \\
\hline & $\mathbf{P}$ & $0.373 * *$ & $0.706^{* *}$ & $-0.225^{* *}$ & 1 & & & & & & & & & \\
\hline \multirow{2}{*}{$\begin{array}{l}\text { Primary } \\
\text { Branches } \\
\text { Per Plant }\end{array}$} & $\mathbf{G}$ & $0.004 \mathrm{NS}$ & $-0.013 \mathrm{NS}$ & $-0.121 N S$ & $-0.075 \mathrm{NS}$ & 1 & & & & & & & & \\
\hline & $\mathbf{P}$ & $0.058 \mathrm{NS}$ & $0.002 \mathrm{NS}$ & $0.041 \mathrm{NS}$ & $-0.063 \mathrm{NS}$ & 1 & & & & & & & & \\
\hline \multirow{2}{*}{$\begin{array}{l}\text { Pods Per } \\
\text { Plant }\end{array}$} & $\mathbf{G}$ & $0.281 * *$ & $0.246^{*}$ & $-0.295 * *$ & $0.337 * *$ & $0.137 N S$ & 1 & & & & & & & \\
\hline & $\mathbf{P}$ & $0.252 *$ & $0.240 *$ & $-0.017 \mathrm{NS}$ & $0.088 \mathrm{NS}$ & $0.324 * *$ & 1 & & & & & & & \\
\hline \multirow{2}{*}{$\begin{array}{c}\text { Pod } \\
\text { Length }\end{array}$} & G & $-0.0048 \mathrm{NS}$ & $0.026 \mathrm{NS}$ & $0.100 \mathrm{NS}$ & $0.096 \mathrm{NS}$ & $0.035 \mathrm{NS}$ & $-0.065 \mathrm{NS}$ & 1 & & & & & & \\
\hline & $\mathbf{P}$ & $-0.027 \mathrm{NS}$ & $0.026 \mathrm{NS}$ & $0.064 \mathrm{NS}$ & $0.058 \mathrm{NS}$ & $-0.090 \mathrm{NS}$ & $-0.070 \mathrm{NS}$ & 1 & & & & & & \\
\hline \multirow{2}{*}{$\begin{array}{l}\text { Seeds Per } \\
\text { Pod }\end{array}$} & G & $0.037 \mathrm{NS}$ & $0.031 \mathrm{NS}$ & $0.065 \mathrm{NS}$ & $0.144 \mathrm{NS}$ & $0.061 \mathrm{NS}$ & $-0.057 \mathrm{NS}$ & $0.995 * *$ & 1 & & & & & \\
\hline & $\mathbf{P}$ & $-0.023 \mathrm{NS}$ & $0.014 \mathrm{NS}$ & $0.064 \mathrm{NS}$ & $0.085 \mathrm{NS}$ & $-0.138 N S$ & $-0.061 \mathrm{NS}$ & $0.942 * *$ & 1 & & & & & \\
\hline \multirow{2}{*}{$\begin{array}{c}\text { Clusters } \\
\text { Per Plant }\end{array}$} & G & $0.173 N S$ & $0.039 \mathrm{NS}$ & $-0.032 \mathrm{NS}$ & $0.000 \mathrm{NS}$ & $-0.136 \mathrm{NS}$ & $-0.341^{* *}$ & $-0.272 * *$ & $-0.233^{* *}$ & 1 & & & & \\
\hline & $\mathbf{P}$ & $0.139 \mathrm{NS}$ & $-0.028 \mathrm{NS}$ & $-0.012 \mathrm{NS}$ & $-0.085 \mathrm{NS}$ & $-0.004 \mathrm{NS}$ & $-0.278 * *$ & $-0.272 * *$ & $-0.225^{*}$ & 1 & & & & \\
\hline \multirow{2}{*}{$\begin{array}{c}\text { Seed } \\
\text { Weight }\end{array}$} & G & $0.473 * *$ & $0.291 * *$ & $-0.083 \mathrm{NS}$ & $0.347 * *$ & $-0.390 * *$ & $0.456^{* *}$ & $-0.123 \mathrm{NS}$ & $-0.046 \mathrm{NS}$ & $-0.018 \mathrm{NS}$ & 1 & & & \\
\hline & $\mathbf{P}$ & $0.401 * *$ & $0.258^{*}$ & $-0.023 \mathrm{NS}$ & $0.329 * *$ & $0.324 * *$ & $0.401 * *$ & $-0.112 \mathrm{NS}$ & $-0.45 \mathrm{NS}$ & $-0.005 \mathrm{NS}$ & 1 & & & \\
\hline \multirow{2}{*}{$\begin{array}{c}\text { Biological } \\
\text { Weight }\end{array}$} & $\mathbf{G}$ & $0.311 * *$ & $0.430 * *$ & $-0.311 * *$ & $0.547 * *$ & $0.245^{*}$ & $0.417 * *$ & $0.009 \mathrm{NS}$ & $0.049 \mathrm{NS}$ & $0.133 \mathrm{NS}$ & $0.639 * *$ & 1 & & \\
\hline & $\mathbf{P}$ & $0.343 * *$ & $0.393^{* *}$ & $-0.183 N S$ & $0.216^{*}$ & $0.525 * *$ & $0.381 * *$ & $0.003 \mathrm{NS}$ & $0.044 \mathrm{NS}$ & $0.117 \mathrm{NS}$ & $0.630 * *$ & 1 & & \\
\hline \multirow{2}{*}{$\begin{array}{l}\text { Harvest } \\
\text { Index }\end{array}$} & $\mathbf{G}$ & $-0.223 *$ & $0.251^{*}$ & $-0.223^{*}$ & $0.316^{* *}$ & $-0.119 \mathrm{NS}$ & $0.128 \mathrm{NS}$ & $0.071 \mathrm{NS}$ & $0.039 \mathrm{NS}$ & $0.263^{*}$ & $-0.233^{*}$ & $0.556 * *$ & 1 & \\
\hline & $\mathbf{P}$ & $0.036 \mathrm{NS}$ & $0.233^{*}$ & $-0.146 \mathrm{NS}$ & $-0.011 \mathrm{NS}$ & $0.305^{* *}$ & $0.131 \mathrm{NS}$ & $0.068 \mathrm{NS}$ & $0.49 \mathrm{NS}$ & $0.291 *$ & $0.230 *$ & $0.538 * *$ & 1 & \\
\hline \multirow{2}{*}{$\begin{array}{c}\text { Seed Yield } \\
\text { per Plant }\end{array}$} & $\mathbf{G}$ & $0.355^{* *}$ & $0.380^{* *}$ & $-0.376^{* *}$ & $0.498 * *$ & $0.138 \mathrm{NS}$ & $0.481 * *$ & $0.074 \mathrm{NS}$ & $0.120 \mathrm{NS}$ & $0.079 \mathrm{NS}$ & $0.497 * *$ & $0.929 * *$ & $0.623 * *$ & 1 \\
\hline & $\mathbf{P}$ & $0.315^{* *}$ & $0.361 * *$ & $-0.238^{*}$ & $0.109 \mathrm{NS}$ & $0.483 * *$ & $0.468^{* *}$ & $0.072 \mathrm{NS}$ & $0.111 \mathrm{NS}$ & $0.063 \mathrm{NS}$ & $0.428 * *$ & $0.592 * *$ & $0.858 * *$ & 1 \\
\hline
\end{tabular}

$\mathrm{G}=$ Genotypic correlation coefficient, $\mathrm{P}=$ Phenotypic correlation coefficient. NS - Non Significant, * Significant at 5\% level, ** Significant at $1 \%$ level 
Table.4 Direct and indirect effects between yield and its related traits in 30 cowpea genotypesat genotypic level and phenotypic levels

\begin{tabular}{|c|c|c|c|c|c|c|c|c|c|c|c|c|c|c|}
\hline Characters & & $\begin{array}{c}\text { Days } \\
\text { to } 50 \% \\
\text { Flower } \\
\text { ing } \\
\end{array}$ & $\begin{array}{l}\text { Days to } \\
\text { 50\% Pod } \\
\text { Setting }\end{array}$ & $\begin{array}{c}\text { Plant } \\
\text { Height } \\
\text { (cm) }\end{array}$ & $\begin{array}{c}\text { Primary } \\
\text { Branches/ } \\
\text { Plant }\end{array}$ & $\begin{array}{l}\text { Days to } \\
\text { Maturity }\end{array}$ & $\begin{array}{l}\text { Pods Per } \\
\text { Plant }\end{array}$ & $\begin{array}{c}\text { Pod } \\
\text { Length } \\
\text { (cm) }\end{array}$ & $\begin{array}{c}\text { Seeds } \\
\text { Per Pod }\end{array}$ & $\begin{array}{l}\text { Clusters } \\
\text { Per Plant }\end{array}$ & $\begin{array}{l}100 \text { Seed } \\
\text { Weight }\end{array}$ & $\begin{array}{l}\text { Biological } \\
\text { yield }\end{array}$ & $\begin{array}{c}\text { Harvest } \\
\text { Index }\end{array}$ & $\begin{array}{l}\text { Correlations } \\
\text { with yield } \\
\text { per plant }\end{array}$ \\
\hline \multirow{2}{*}{$\begin{array}{c}\text { Days to 50\% } \\
\text { Flowering }\end{array}$} & G & 0.0360 & -0.0180 & 0.0127 & -0.0005 & -0.1069 & 0.0253 & -0.0643 & 0.04731 & 0.00163 & 0.08337 & 0.3350 & 0.0031 & $0.355^{* *}$ \\
\hline & $\mathbf{P}$ & 0.0043 & 0.04708 & -0.01009 & -0.00199 & 0.01128 & 0.0484 & 0.0032 & -0.0045 & 0.001 & -0.0660 & 0.2802 & 0.0025 & $0.315^{* *}$ \\
\hline \multirow{2}{*}{$\begin{array}{c}\text { Days to } 50 \% \\
\text { Pod Setting }\end{array}$} & G & 0.0717 & -0.00623 & 0.03701 & 0.00198 & -0.19912 & 0.0221 & 0.0346 & -0.03973 & -0.00037 & 0.05126 & 0.3724 & 0.03446 & $0.380^{* * *}$ \\
\hline & $\mathbf{P}$ & 0.0136 & 0.01585 & -0.02997 & -0.00006 & 0.02136 & 0.04615 & -0.0038 & 0.00289 & -0.0002 & -0.04247 & 0.32117 & 0.0161 & $0.361 * *$ \\
\hline \multirow{2}{*}{$\begin{array}{l}\text { Plant Height } \\
\text { (cm) }\end{array}$} & G & -0.1921 & 0.0033 & -0.0138 & 0.0182 & 0.098 & -0.0265 & 0.135 & -0.0826 & -0.0003 & -0.0147 & -0.2697 & -0.0305 & $-0.376^{* *}$ \\
\hline & $\mathbf{P}$ & -0.0515 & -0.0039 & 0.0079 & 0.0014 & -0.0068 & -0.034 & -0.0077 & 0.013 & $-8 \mathrm{E}-05$ & 0.0038 & -0.1495 & 0.0025 & $-0.238^{*}$ \\
\hline \multirow{2}{*}{$\begin{array}{c}\text { Primary } \\
\text { Branches/Plant }\end{array}$} & G & 0.0233 & -0.00007 & -0.0004 & -0.15061 & 0.0199 & 0.01229 & 0.0477 & -0.07785 & -0.00128 & 0.06869 & 0.2123 & -0.01635 & $0.138 \mathrm{NS}$ \\
\hline & $\mathbf{P}$ & 0.0021 & 0.0027 & -0.00005 & -0.03448 & -0.0019 & 0.01696 & -0.0070 & 0.01717 & -0.00061 & -0.05414 & 0.17638 & -0.0080 & $0.483^{* * *}$ \\
\hline \multirow{2}{*}{$\begin{array}{c}\text { Days to } \\
\text { Maturity }\end{array}$} & G & 0.0705 & -0.0072 & 0.0275 & 0.0112 & -0.2670 & 0.0303 & -0.1290 & 0.1833 & 0 & 0.06121 & 0.4737 & 0.0433 & $0.498^{* * *}$ \\
\hline & $\mathbf{P}$ & 0.0116 & 0.0175 & -0.02115 & 0.0021 & 0.0302 & 0.0624 & 0.0108 & -0.0280 & -0.00003 & -0.0533 & 0.4293 & 0.0211 & $0.109 \mathrm{NS}$ \\
\hline \multirow[t]{2}{*}{ Pods Per Plant } & G & 0.0567 & -0.0050 & 0.0090 & -0.0205 & -0.09008 & 0.090 & -0.0877 & 0.07216 & -0.00322 & 0.0803 & 0.3614 & 0.0175 & $0.481 * *$ \\
\hline & $\mathbf{P}$ & 0.0091 & 0.0118 & -0.0071 & -0.00304 & 0.00981 & 0.1925 & 0.008 & -0.01242 & -0.00201 & -0.0660 & 0.3180 & 0.0091 & $0.468^{* *}$ \\
\hline \multirow{2}{*}{$\begin{array}{l}\text { Pod Length } \\
\text { (cm) }\end{array}$} & G & -0.019 & 0.0008 & 0.0009 & -0.0053 & 0.0255 & -0.0058 & 1.3508 & -1.26681 & -0.00256 & -0.0217 & 0.0075 & 0.0096 & $0.074 \mathrm{NS}$ \\
\hline & $\mathbf{P}$ & -0.0033 & -0.0012 & -0.0007 & -0.00201 & -0.0027 & -0.0135 & -0.1204 & 0.1913 & -0.00197 & 0.02003 & 0.0023 & 0.0047 & $0.072 \mathrm{NS}$ \\
\hline \multirow[t]{2}{*}{ Seeds Per Pod } & G & -0.0124 & 0.0006 & 0.0011 & -0.0092 & 0.0384 & -0.0051 & 1.3434 & -1.2738 & -0.0022 & -0.00814 & 0.0421 & 0.0052 & $0.120 \mathrm{NS}$ \\
\hline & $\mathbf{P}$ & -0.0032 & -0.0010 & -0.0004 & -0.0029 & -0.0041 & -0.0117 & -0.1134 & 0.2030 & 0.0016 & 0.00742 & 0.0360 & 0.0034 & $0.111 \mathrm{NS}$ \\
\hline \multirow{2}{*}{$\begin{array}{c}\text { Clusters Per } \\
\text { Plant }\end{array}$} & G & 0.0062 & -0.0031 & -0.0014 & 0.0204 & 0.00009 & -0.0306 & -0.3669 & 0.29725 & 0.0094 & -0.0031 & 0.1149 & 0.0360 & $0.079 \mathrm{NS}$ \\
\hline & $\mathbf{P}$ & 0.0006 & 0.0065 & 0.0008 & 0.0029 & -0.0001 & -0.0535 & 0.0327 & -0.0456 & 0.0072 & 0.0008 & 0.0956 & 0.0151 & $0.063 \mathrm{NS}$ \\
\hline \multirow{2}{*}{$\begin{array}{l}100 \text { Seed } \\
\text { Weight }\end{array}$} & G & 0.0160 & -0.0085 & 0.0107 & -0.0587 & -0.0927 & 0.0410 & -0.1663 & 0.0588 & -0.0001 & 0.1762 & 0.5530 & -0.0319 & $0.497 * *$ \\
\hline & $\mathbf{P}$ & 0.0012 & 0.0189 & -0.0077 & -0.0113 & 0.0098 & 0.0772 & 0.0146 & -0.00914 & -0.00004 & -0.1646 & 0.5153 & -0.0159 & $0.428^{* *}$ \\
\hline \multirow[t]{2}{*}{ Biological yield } & G & 0.0598 & -0.0069 & 0.0159 & -0.03693 & -0.14612 & 0.0375 & 0.0117 & -0.06201 & 0.0012 & 0.1125 & 0.4814 & 0.13715 & $0.929^{* * *}$ \\
\hline & $\mathbf{P}$ & 0.0075 & 0.0017 & -0.0069 & 0.004 & 0.00923 & 0.0252 & -0.008 & 0.01 & 0.0015 & 0.03786 & 0.4402 & 0.0693 & $0.592 * *$ \\
\hline \multirow{2}{*}{ Harvest Index } & G & 0.0428 & -0.0004 & 0.0093 & 0.0179 & -0.0843 & 0.0115 & 0.0952 & -0.0491 & 0.0024 & -0.0410 & 0.8659 & 0.0762 & $0.623^{* *}$ \\
\hline & $\mathbf{P}$ & 0.0094 & 0.0161 & -0.0117 & -0.0074 & 0.0158 & 0.0748 & -0.0003 & -0.0089 & 0.0008 & -0.1037 & 0.8178 & 0.0373 & $0.858^{* *}$ \\
\hline
\end{tabular}

Bold diagonal are direct effects; G: Genotypic Path coefficient, Residual effect (G):0.10550; P: Phenotypic Path coefficient, (P): 0.19624 
The correlation values provided only nature and degree of relationship of yield contributing characters on seed yield. In the present study path coefficient analysis was carried out using genotypic and phenotypic correlation matrix of 13 characters (Table 4). Path values revealed that days to $50 \%$ flowering, pods per plant, clusters per pod, harvest index, biological yield showing direct positive effect on seed yield at both phenotypic and genotypic levels. Primary branches per plant showed direct and negative effect on seed yield at both phenotypic and genotypic levels, but it contributes to yield indirectly through the number of pods per plant, biological yield, days to $50 \%$ flowering. Pod length, 100 seed weight, showed direct and positive effect on seed yield at genotypic level. Days to $50 \%$ maturity, days to $50 \%$ pod setting, plant height, seeds per pod showed direct positive effect on seed yield at phenotypic level. Plant height, days to 50\% pod setting, days to maturity and seeds per plant showed negative direct effect on seed yield at genotypic level but the days to 50\% pod setting, days to maturity shows positive significant genotypic correlation with seed yield it indicates these traits are contributing indirectly to the seed yield. Most of these results are in accordance with the previous workers (Patel et al., 2016; Suganthi et al., 2008). These results indicate that the selection of these traits such as days to $50 \%$ flowering, days to $50 \%$ pod setting, pods per plant, 100 seed weight, biological yield may improve seed yield.

\section{References}

AE, H., Ismail, A. M., Ehlers, J. D., Marfo, K. O., Cisse, N., Thiaw, S., and Close, T. J. (2002). Breeding cowpea for tolerance to temperature extremes and adaptation to drought. Challenges and opportunities for enhancing sustainable cowpea production, 14 .
Al-Jibouri, H., Miller, P. A., and Robinson, H. F. (1958). Genotypic and Environmental Variances and Covariances in an Upland Cotton Cross of Interspecific Origin 1. Agronomy journal, 50(10), 633-636.

Ananomus. 2011. Annual report of Indian Council of Medical Research, Hyderabad.

Burton, G. W., and Devane, E. H. (1953). Estimating heritability in tall fescue (Festuca arundinacea) from replicated clonal material 1. Agronomy Journal, 45(10), 478-481.

Chopra, V. L., and Prakash, S. (2002). Evolution and adaptation of cereal crops. Science Publishers, Inc.

Dewey, D. R., and Lu, K. (1959). A Correlation and Path-Coefficient Analysis of Components of Crested Wheatgrass Seed Production 1. Agronomy journal, 51(9), 515-518.

Fisher, R. A. (1936). Design of experiments. Br Med J, 1(3923), 554-554.

Hair, J. F., Black, W. C., Babin, B. J., Anderson, R. E., and Tatham, R. L. (2006). Multivariate data analysis (Vol. 6).

Hamdi, A. (1992). Heritability and combining ability of root characters in lentil (Lens culinaris Medik.). Egyptian Journal of Agricultural Research (Egypt).

Hazra, P. and Som, M.G.(1999). Technology for vegetable production and improvement, ISBN 81-85421-44-7.19.

Johnson, H. W., Robinson, H. F., and Comstock, R. E. (1955). Estimates of genetic and environmental variability in soybeans 1. Agronomy journal, 47(7), 314-318.

Lal, H., Rai, M., Karan, S., Verma, A., and Ram, D. (2006, December). Multivariate hierarchical clustering of cowpea germplasm (Vigna unguiculata (L.) Walp. In I International Conference on Indigenous Vegetables 
and Legumes. Prospectus for Fighting Poverty, Hunger and Malnutrition 752(pp. 413-416).

Lush, J. L., Lamoreux, W. F., and Hazel, L. N. (1948). The heritability of resistance to death in the fowl. Poultry Science, 27(4), 375-388.

Nigude, A. D., Dumbre, A. D., Sushir, K. V., Patil, T. F. E., and Chavhan, A. D. (2004). Correlation and path coefficient analysis in cowpea. Annals of Plant Physiology, 18(1), 71-75.

Pal, A.K., Maurya, A.N., Singh, B., Ram, D. and Kumar, S. (2003). Genetic variability, heritability and genetic advance in cowpea [Vigna unguiculata (L.) Walp]. Orissa J. Hort., 31(1): 9497.

Patel, U. V., Parmar, V. K., Patel, P. B., and Malviya, A. V. (2016). Correlation and Path Analysis Study in Cowpea (Vigna unguiculata (L.) Walp.). International Journal of Science, Environment and Technology, 5(6), 3897-3904.

Singh, C. (1983). Modern Techniques of Raising Field Crops. Oxford and IBH Publishing Company, New Delhi, pp. 237-244.

Singh, N. and Singh, V.P. (1997). Studies on character association and path coefficient analysis in cowpea (Vigna unguiculata L.). Annals of Agricultural Bio Research, 2: 43-47.

Suganthi, S., and Murugan, S. (2008). Association analysis in cowpea (Vigna unguiculata L. Walp). Legume Research-An International Journal, 31(2), 130-132.

Tarawali, S.A., Singh, B.B., Kormawa, P.M. and Tamo, M. (eds.), Intl Inst Tropical Agric, Ibadan, Nigeria, pp 14-21.

\section{How to cite this article:}

Nandini Priya Yadav, B. and Duddukur Rajasekhar. 2019. Genetic Variability, Trait Association and Path Analysis in Cowpea (Vigna unguiculata L. Walp). Int.J.Curr.Microbiol.App.Sci. 8(12): 1979-1986. doi: https://doi.org/10.20546/ijcmas.2019.812.236 